\title{
RETRACTED ARTICLE: Formal derivation of biconjugate gradient method with its modification for distributed parallel computing
}

\author{
Tong-Xiang Gu • Jia-Min Wang • Xian-Yu Zuo • \\ Bing Yang • Xing-Ping Liu
}

Received: 1 April 2013 / Accepted: 6 March 2014 / Published online: 22 October 2014

(C) Springer Science+Business Media Dordrecht 2014

Upon investigation carried out according to the Committee on Publication Ethics guidelines, it has been found that the authors of this article had duplicated with only minor changes and inadequate acknowledgment significant parts of the following articles:

D'Azevedo, E F, Eijkhout, V L and Romine, C H (1993) LAPACK Working Note 56: Conjugate Gradient Algorithms with Reduced Synchronization Overhead on Distributed Memory Multiprocessors, Technical Report CS-93-185, Department of Computer Science, University of Tennessee, 1993

D'Azevedo, E, Eijkhout, V and Romine, C (1993) A Matrix Framework for Conjugate Gradient Methods and Some Variants of CG with Less Synchronization Overhead, Proceedings of the Sixth SIAM Conference on Parallel Processing for Scientific Computing, SIAM, Philadelphia, 1993, pp. 644-646

Eijkhout, V, Bientinesi, P and van de Geijn, R (2007) Applying Formal Derivation Techniques to Krylov Subspace Methods, TACC Technical Report TR-07-02, Texas Advanced Computing Center, The University of Texas at Austin, 2007

\footnotetext{
T.-X. Gu (凶) · X.-P. Liu

Laboratory of Computational Physics, Institute of Applied Physics and Computational Mathematics,

P.O. Box 8009, Beijing 100088, People's Republic of China

e-mail: gu_tx@sina.com

J.-M. Wang

Institute of Electromagnetics and Acoustics, Xiamen University, Xiamen 361005, People's Republic of China

B. Yang

Graduate School of Chinese Academy of Engineering Physics, P.O. Box 2101,

Beijing 100088, People's Republic of China

X.-Y. Zuo

College of Computer and Information Engineering, Henan University,

Kaifeng 475004, People's Republic of China
} 